\title{
Wound fluids collected from patients after IORT treatment activates extrinsic apoptotic pathway in MCF7 breast cancer cell line
}

\author{
Katarzyna Ida Kulcenty1, 2 , Igor Piotrowski', 2, Karolina Zaleska', Dawid Murawa³, \\ Wiktoria Maria Suchorska ${ }^{1,2}$ \\ ${ }^{1}$ Radiobiology Labolatory, Department of Medical Physics, Greater Poland Cancer Centre, Poznan, Poland \\ ${ }^{2}$ Department of Electroradiology, University of Medical Sciences, Poznan, Poland, \\ ${ }^{3}$ Department of General and Minimally Invasive Surgery, Poland Baptism Monument Hospital, Gniezno, Poland
}

\begin{abstract}
Objectives: Intraoperative radiotherapy (IORT) relates to irradiation of diseased tissue during the surgery within the tumor bed. The reason for this process is based on the fact that the increase in the radiation dose increases local tumor control. It was shown that postoperative fluids obtained from patients after breast cancer conserving surgery, stimulated motility and invasiveness of tumor cells in vitro. The results obtained from TARGIT clinical trial demonstrated that IORT significantly inhibits the stimulatory effect of wound fluids on tumor cells in vitro. We therefore speculated that wound fluids collected from patients after IORT treatment may induce the apoptosis in breast cancer cell lines and it may be a reason for their lower proliferation rate and potential to metastasis.

Material and methods: Breast cancer MCF7 cell line was incubated with wound fluids collected from patients after conserving breast cancer surgery or surgery followed by IORT for 4 days. Then the expression of markers associated with extrinsic or intrinsic apoptosis pathway was established.

Results: Our results clearly indicate activation of extrinsic apoptosis pathway by wound fluids collected from patients after IORT treatment. No changes in apoptotic markers were seen in cells treated with wound fluids collected from patients after the surgery alone.

Conclusions: Thus we confirmed that wound fluids collected from patients after IORT treatment may induce the apoptosis in breast cancer cell lines and it may be a reason for their lower proliferation rate and invasiveness of tumor cells in vitro.

Key words: apoptosis, wound fluids, intraoperative radiation therapy, breast cancer
\end{abstract}

Ginekologia Polska 2018; 89, 4: 175-181

\section{INTRODUCTION}

The development of normal tissue depends on the balance between cell proliferation and apoptosis. It is believed that the growth of the tumor is not only caused by uncontrollable proliferation of cells but also by reduced apoptosis rate. It is speculated that low levels of apoptosis are characteristic for a poor prognosis in breast cancer. Nevertheless many research show that programmed cell death is often increased in malignant tumors, however it is then often followed by high levels of proliferation [1]. Increased resistance of human breast cancer cells to apoptosis is induced by high expression of IL-6 and IL-6R. Moreover, as shown by Wang et al. IL-17 can suppress apoptosis and promote tumor proliferation, survival and metastasis by upregulating the expression of VEGF, MMP2 and MMP9 [2]. It has been proven that surgery and especially wound fluids secreted from tissue after the surgery induces inflammatory response [3]. Moreover until recently a hypothesis has been made, that external inflammatory pathways promote or even initiate cancer [4]. Many studies revealed that approximately $90 \%$ of local recurrences in the breast after surgery appears in the same quadrant as the primary cancer [5]. The published data 
suggest that this process of recurrence is caused by wound healing process which alters the tumor microenvironment thus leading the tumor to be more favorable to recur [3].

Intraoperative radiotherapy (IORT) relates to irradiation of diseased tissue during the surgery within the tumor bed [6]. The reason for this process is based on the evidence that the increase in the radiation dose increases local tumor control. Studies of Belletti and Tagliabule [7, 8] have shown that postoperative fluids obtained from patients after breast cancer conserving surgery stimulated motility and invasiveness of tumor cells in vitro. The results obtained from TARGIT clinical trial demonstrated that IORT significantly inhibits the stimulatory effect of WF on tumor cells in vitro [7]. This effect may be due to both direct cell killing by ionizing radiation and by the modulation of tumor microenvironment [9]. It was proved that during the procedure of IORT, the cells produce specific cytokines and chemokines that affect non-irradiated cells [10]. Those molecules produced in the response to irradiation may fulfill both pro- and anti-apoptotic functions in cancer. Thus we speculated, that wound fluids after IORT may induce the apoptosis in breast cancer cells and it may be a reason for a lower proliferation and capability to metastasis.

Apoptosis is a conserved program of controlled cell death, that is seen in various physiological and pathological situations [11]. It is believed that cancer cells are able to evade apoptosis and thus mostly the anticancer therapies results in the activation of apoptosis pathway mainly by activation of caspases [12]. Activation of caspases activity is initiated both at the plasma membrane level by death receptors (receptor = extrinsic pathway) and at a mitochondrial level (mitochondrial = intrinsic pathway) [11]. While the activation of extrinsic apoptosis pathway is dependent on stimulation of death receptors by external stimuli, the activation of intrinsic pathway is a result of intracellular stimuli like for example DNA damage [13].

\section{Objectives}

The aim of this research is to analyze the effect of post-surgical wound fluids after IORT treatment on the expression of the markers associated with apoptosis. We believe the findings reported here will contribute to better understanding of biological processes involved in response of breast cancer cells to IORT treatment and will be particularly relevant in the effectiveness of breast cancer treatment.

\section{MATERIAL AND METHODS Surgical wound fluids}

Wound fluids were collected from patients 7 days after breast cancer surgery by percutaneous aspiration. They were divided into two groups. One group consisted of patients, which underwent breast cancer conserving surgery (quadranectomy) (WF group; age at diagnosis $=50 \pm 11.4$, ER status:
100\% ER positive, HER status: 0\% HER2 positvie, histopathological status: $100 \%$ ductal). The second group consisted of patients, in whom the surgery was followed by intraoperative radiation therapy given as a boost of $10 \mathrm{~Gy}$ (RT-WF group; age at diagnosis $=55.8 \pm 10,03$, ER status: $100 \%$ ER positive, HER status: 0\% HER2 positvie; histopathological status: $100 \%$ ductal). Wound fluids were collected as described elsewhere [14]. Shortly fluids were collected by percutaneous aspiration, centrifuged for $25 \mathrm{~min}$ at $300 \times \mathrm{g}$ at $4^{\circ} \mathrm{C}$, sterile filtered and stored at $-80^{\circ} \mathrm{C}$. This study was approved by the Bioethics Committee of Poznan University of Medical Sciences number 756/16.

\section{Cell culture}

A luminal subtype of breast cancer cell line - MCF7 (Er/PgR+; HER2/Neu-) was obtained from the American Type Culture Collection (ATCC, Rockville, MD) and cultured according to ATCC instructions in a humidified atmosphere with $5 \%$ carbon dioxide and $37^{\circ} \mathrm{C}$ (BINDER, Germany). Cells were incubated with $10 \%$ of WF or RT-WF in Dulbecco's Modified Eagle's Medium (DMEM, BIOWEST, UT) with $10 \%$ fetal bovine serum (FBS, BIOWEST, UT) for 4 days and then subjected to RNA isolation. Control cells were cultured in standard medium (10\% FBS in DMEM) under the same conditions. This cell line was chosen based on ER/PR.HER2 status of patients from each wound fluids were collected. Based on the receptor expression, MCF7 cell line corresponds the best with patient's cancer status.

\section{RNA isolation and RT-PCR}

Total RNA was isolated using TRI Reagent (Sigma-Aldrich, $\mathrm{MI})$, according to the manufacturer's instructions. The RNA was eluted in DEPC-treated $\mathrm{H}_{2} \mathrm{O}$ and stored at $-80^{\circ} \mathrm{C}$ until further analyses. $1 \mu \mathrm{g}$ of RNA was then subjected to reverse transcription using iSCRIPT kit (BioRad, CA) according to manufacturer's instructions.

\section{Real-time quantitative PCR (RT-qPCR)}

The expression of markers associated with intrinsic and extrinsic apoptosis pathway was analyzed using RealTime UPL Probes Master system (Roche, Basel, Switzerland) on LightCycler96 (Roche, Basel, Switzerland). The expression of analyzed genes was normalized to the expression of beta actin reference gene. The fold-differences in genes expression were calculated using the comparative $\mathrm{Cq}(\Delta \Delta \mathrm{Cq})$ method. Quantitative differential expression was calculated as $2^{-\Delta \Delta C q}$ [15].

\section{Statistical analysis}

Statistical analysis was performed using GraphPad Prism (GraphPad Software, CA). Data were examined using the One-Way ANOVA with Tukey post hoc test and differences were considered significant at $\mathrm{p}<0.05$. Data are displayed as mean $\pm \mathrm{SD}$. 


\section{RESULTS \\ Effects of WF and RT-WF on the induction of apoptosis pathway}

To study a potential effect of surgical wound fluids after intraoperative radiotherapy on the induction of extrinsic and intrinsic apoptosis pathway, human breast cancer cells (MCF7 cell line) were incubated for 4 days either with WF or RT-WF. Then the expression level of genes associated with extrinsic and intrinsic apoptosis pathway was assessed by RT-qPCR.

\section{Extrinsic apoptosis pathway}

The extrinsic apoptosis pathway is associated with the activation of CASP8 or CASP10 by binding of a ligand to cell surface receptors. The expression of genes involved in extrinsic apoptosis pathway was assessed by RT-qPCR in MCF7 cell line stimulated with WF or RT-WF. The untreated cells were used as a control. A highly significant increase in the expression of TNF (tumor necrosis factor) and TRAIL (TNF-related apoptosis-inducing factor) was observed after stimulation with RT-WF (TNF $=3.51 \pm 0.02$, TRAIL $=8.15 \pm 2.08$ ) while comparing to untreated cells (CTR) (TNF $=0.78 \pm 0.3$, TRA$\mathrm{IL}=0.918 \pm 0.117$ ). No statistically significant changes in the expression of those markers were seen in WF stimulated group $($ TNF $=0.38 \pm 0.03$, TRAIL $=2.96 \pm 0.61)$. The TNF receptor family has more than 20 receptors. They activate apoptosis by a cytoplasmic domains that recruit the death domain containing adaptor FAS-associated via death domain (FADD) protein. Then FADD recruits CASP8 and/or 10 which in turn results in the activation of apoptosis pathway [16]. Therefore, the expression of two death receptors as well as FASS and CASP8 and CASP10 was analyzed. An increase in the expression of two TNF receptors:TNFRSF10A (DR4) and TNFRSF10B (DR5) was observed in RT-WF treated MCF7 cells (TNFRS$F 10 A=1.47 \pm 0.16$, TNFRSF10B $=1.92 \pm 0.13$ ) comparing to CTR (TNFRSF10A $=1.01 \pm 0.0001$, TNFRSF10B $=1.13 \pm 0.18)$. No significant changes in WF treated group were observed (TNFRSF10A $=1.01 \pm 0.0001$, TNFRSF10B $=1.38 \pm 0.04$ ). On the contrary to previously mentioned markers of extrinsic pathway of apoptosis, FADD expression was statistically decreased in WF treated cells $(0.853 \pm 0.008)$ comparing to CTR $(1 \pm 0.00001)$. While a significant increase was observed for CASP10 expression in RT-WF group $(5.38 \pm 0.009)$ compared to both CTR $(1.19 \pm 0.27)$ and WF $(2.11 \pm 0.80)$, no significant change in CASP8 activity was observed. (CTR $=0.984 \pm 0.065$, $\mathrm{WF}=0.958 \pm 0.14, \mathrm{RT}-\mathrm{WF}=1.2 \pm 0.24)($ Fig. 1)

\section{Intrinsic apoptosis pathway}

The expression of 7 genes involved in extrinsic apoptosis pathway was assessed by RT-qPCR in MCF7 cell line stimulated with WF or RT-WF. The untreated cells were used as a control. In the intrinsic (mitochondrial) apoptosis pathway, the activation of caspase activity is related to permeabilization of the outer mitochondrial membrane by the Bcl family members. In the MCF7 cell line treated with WF and RT-WF a significant decrease in $\mathrm{BCl}-2$ expression was observed in WF treated cells $(0.201 \pm 0.007)$ comparing both to CTR $(0.993 \pm 0.01)$ and RT-WF group $(0.863 \pm 0.095)$. Upon disruption of the outer mitochondrial membrane, cytochrome $\mathrm{C}$ is released which results in the formation of cytochrome c/APAF-1/CASP9 apoptosome complex which in turn triggers CASP3, CASP6 and CASP9 activation. While in analyzed MCF7 cell line no change in APAF-1 expression was observed in any of analyzed groups $(C T R=1.04 \pm 0.06, W F=1.1 \pm 0.15$, RT-WF $=1.17 \pm 0.34)$, the CASP9 was slightly increased in both WF $(1.33 \pm 0,1)$ and RT-WF treated cells $(1.18 \pm 0.04)$ compared to CTR $(0.99 \pm 0.01)$. From the other caspase family members, only decrease in CASP3 activity was observed in WF treated cells $(0.648 \pm 0.07)$ compared both to CTR (1.0 \pm 0.0001$)$ and RT-WF treated cells (0.818 \pm 0.177$)$. DIABLO expression also did not change after any of wound fluids stimuli $(C T R=0.954 \pm 0.065, W F=0.967 \pm 0.057, R T-$ $-W F=0.996 \pm 0.211$ ) (Fig. 2)

\section{DISCUSSION}

The wound healing process after breast cancer conserving surgery activates inflammatory responses, which are known to modify tumor microenvironment and growth kinetics of breast cancer metastasis $[3,17]$. Those results suggest, that wound fluids can be a significant factor in a local recurrence or development of metastasis. As proved by Belletti et al. [7] cytokines and growth factors present in the wound fluids are involved in the stimulation of mammary carcinoma cells. Their work confirms that wound fluids harvested from breast cancer patients, who underwent conserving breast cancer surgery, triggers the motility of breast cancer cells, their invasion and growth in three-dimensional culture. Authors also prove, that this effect may be partially abrogated by wound fluids collected from patients after IORT treatment. The inhibitory effect of wound fluids collected from breast cancer patients after IORT on the proliferation was also confirmed by Veldwijk et al. [18]. These findings are consistent with the published data, showing that IORT treatment changes the tumor microenvironment by modulating the concentration of cytokines in wound fluids. IL- 6 is one of the cytokines whose concentration is significantly decreased in wound fluids collected from patients after IORT [7]. Upon binding to its membrane receptor, it activates JAK/STAT3 signaling pathway. Consistent with these findings, Segatto et al. confirmed that STAT3 was highly activated in breast cancer cells following stimulation with wound fluids [19]. While IL-6 activity is much decreased in IORT treated patients, thus they assumed and confirmed afterwards, that breast cancer cells treated with wound 

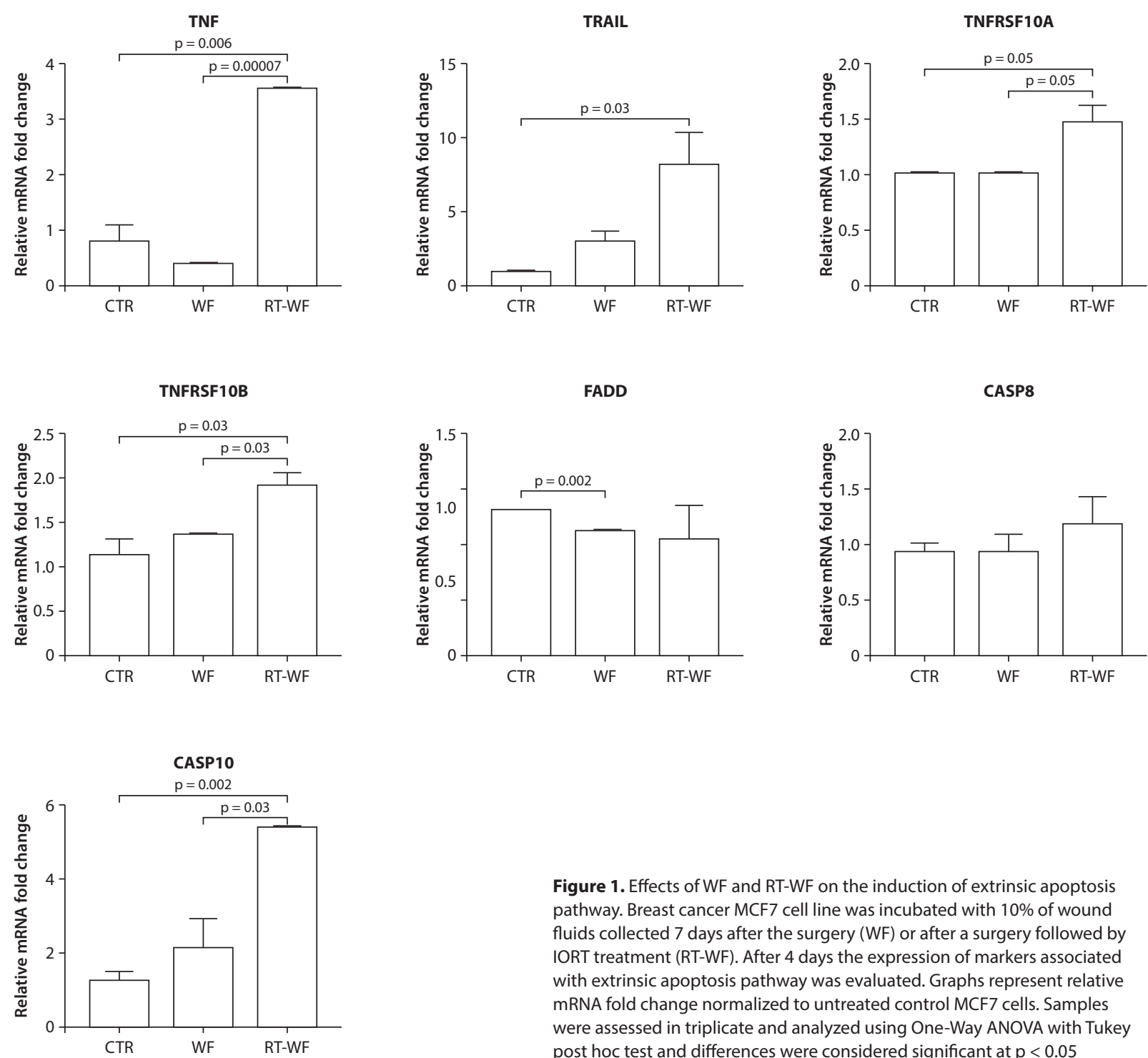

Figure 1. Effects of WF and RT-WF on the induction of extrinsic apoptosis pathway. Breast cancer MCF7 cell line was incubated with $10 \%$ of wound fluids collected 7 days after the surgery (WF) or after a surgery followed by IORT treatment (RT-WF). After 4 days the expression of markers associated with extrinsic apoptosis pathway was evaluated. Graphs represent relative mRNA fold change normalized to untreated control MCF7 cells. Samples were assessed in triplicate and analyzed using One-Way ANOVA with Tukey post hoc test and differences were considered significant at $p<0.05$

fluids collected from IORT patients show lower proliferation rate, than cells incubated with wound fluids after the surgery only. They also showed, that the suppression of STAT3 activity in breast cancer cells significantly decreased the growth of tumor in STAT3-silenced mouse model. STAT3 activity is stimulated by IL- 6 . All of these factors can affect proliferation of the breast cancer cells, however questions have been raised concerning the activation of death pathway in RT-WF treated cells. Based on these results, we postulated that decreased proliferation capability of breast cancer cell lines stimulated with RT-WF may be caused by activation of apoptotic pathway in those cells. Thus we incubated MCF7 cell line with WF and RT-WF and analyzed the transcriptomic activation of apoptosis. We analyzed both the markers of extrinsic and intrinsic apoptosis pathway. The findings presented in this study indicate, that extrinsic pathway of apoptosis is activated in response to RT-WF stimulation of MCF7 cell line. The expression of genes involved in the different stages of apoptosis pathway were highly enriched in RT-WF group compared to CTR and WF stimulated cells. Likewise, we did not observe any changes in the expression of genes involved in intrinsic apoptosis pathways after RT-WF stimulation comparing to untreated cells. An opposite results are observed in WF stimulated MCF7 cell line. The expression of both extrinsic and intrinsic apoptosis markers was not changed or slightly decreased in response to WF. These findings provide valuable data on the activation of apoptosis pathway by external stimuli in RT-WF group. Moreover, they are supported by the fact previously described by Belletti et al. [7], that IORT changes the proteomic profile of wound fluids, which results in different biological activities of RT-WF and WF observed in proliferation and motility assays. Based on our data, we can speculate, that wound fluids collected from the patients after IORT treatment affect the proliferation of 
BCL2

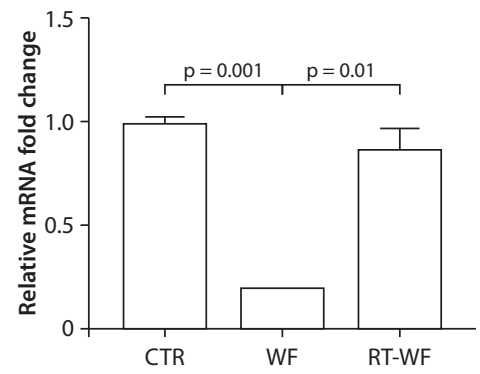

CASP3

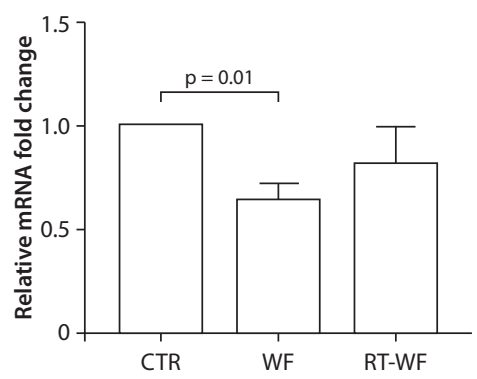

DIABLO

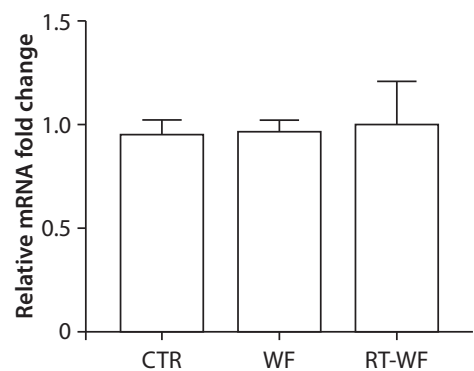

APAF-1

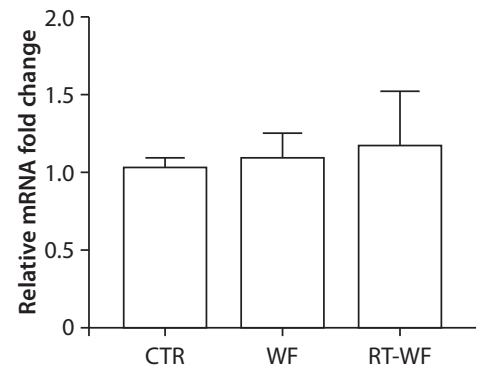

CASP6

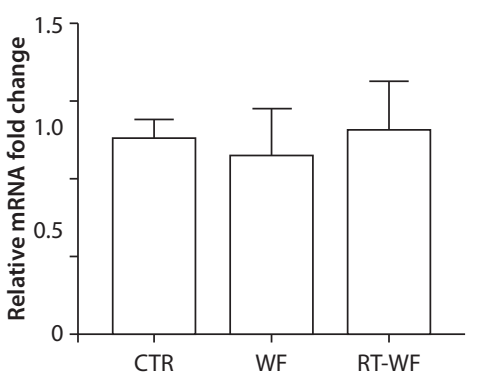

CASP9

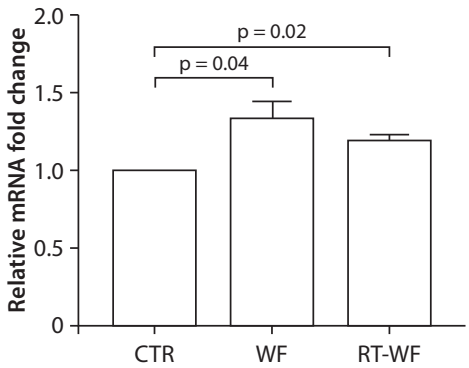

CASP7

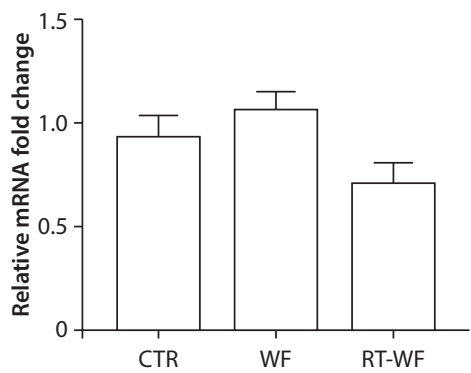

Figure 2. Effects of WF and RT-WF on the induction of intrinsic apoptosis pathway. Breast cancer MCF7 cell line was incubated with $10 \%$ of wound fluids collected 7 days after the surgery (WF) or after a surgery followed by IORT treatment (RT-WF). After 4 days the expression of markers associated with intrinsic apoptosis pathway was evaluated. Graphs represent relative mRNA fold change normalized to untreated control MCF7 cells. Samples were assessed in triplicate and analyzed using One-Way ANOVA with Tukey post hoc test and differences were considered significant at $p<0.05$

MCF7 cell line by inducing apoptosis in those cells. Higher proliferation and motility of breast cancer cells after WF stimulation may be due to lack of apoptosis induction, thus leading to higher proliferation rate.

It has been proven that killing of the cancer cells in cancer therapy has been associated with the activation of apoptosis pathway [20]. A critical component in apoptotic cell death plays activation of proteolytic enzymes known as caspases. Many signaling pathways activated in anticancer therapies result in activation of caspases. Depending on the type of apoptotic pathway, different caspases are being activated. The extrinsic apoptosis pathway results mainly in activating CASP8 or/and CASP10, while intrinsic apoptosis pathway results in activation of CASP3/6/9. While the caspases are key components of apoptotic pathway, many anticancer therapies focus on targeting upstream molecules of apoptotic pathway. Many studies investigated a potential role of TRAIL in combination with chemotherapeutic drugs in order to intensify cell death and/or overcome resistance to common treatment [21,22]. Concomitant administration of TRAIL and the chemotherapeutic drugs caused significant activation of caspases [21]. TRAIL is an inducer of extrinsic apoptosis pathway that acts through a complicated receptor system. Based on the selectivity of the soluble recombinant TRAIL towards transformed cells, this molecule is a promising therapeutic agent in cancer. As mentioned by Wang et al. identifying new agents that target death receptors may be useful for cancer therapy [23]. Experiments on breast cancer cell lines suggested that they are very resistant to TRAIL-induced apoptosis. In addition in many of them this effect may be overcome by concomitant administration of chemo- or radiotherapy [24]. According to Ashkenazi et al. administration of recombinant soluble TRAIL in mouse model significantly induces tumor regression without 
systemic toxicity [25]. Moreover, it is important to point out that TRAIL induces apoptosis regardless of p53 status and thus has a high ability to kill cancer cells with the mutation in the p53 gene [26]. As shown by Chinnaiyan et al. radiation intensifies TRAIL-mediated apoptosis in both cell lines and tumor xenografts [27]. There is evidence that ER expressing cell lines are resistant to the induction of apoptosis by TRAIL alone $[21,22,28]$. Those authors found out that synergistic interaction between TRAIL and chemotherapeutic drugs can overcome TRAIL resistance in ER positive breast cancer cells. A study by Lagadec et al. [29] showed that tamoxifen can intensify TRAIL-induced apoptosis in breast cancer cells both in vitro and in vivo. Based on our data we believe that IORT treatment can overcome the TRAIL-dependent resistance to apoptosis. Nevertheless, given the preliminary nature of the current study, more research is needed to reach definitive conclusions.

The induction od DR5 expression is very important in TRAIL induced apoptosis [26]. Thus targeting this receptor may be a promising factor in cancer therapy. Wang et al. also pointed out that upregulation of DR5 in tumors that retain some responsiveness to therapy might lead to the activation of apoptosis and reduce the probability of tumor cells to be resistant to treatment [26]. We confirmed that DR5 expression is significantly induced in RT-WF treated cells. While the patients after IORT treatment may also get concomitant radiotherapy after the surgery, based on our results we can assume, that activation of apoptosis may sensitize them for further radiation treatment comparing to patients which underwent surgery alone. However this conclusion is not without risk, given that apoptosis is a very complex process and more research is needed to reach definitive conclusions.

Another important aspect in cancer therapy is induction ofTRAIL-dependent apoptosis together with histone deacetylase (HDAC) inhibitors. Many cancers display increased expression of HDAC, which thus results in condensation of chromatin and downregulation of genes associated with tumor suppression. The HDAC inhibitors used as anticancer therapy show promising effects in both pre-clinical and clinical setting [30] by inducing cell cycle arrest, promoting differentiation, and causing cancer death [31]. Moreover HDAC inhibitors are shown to be an efficient radiosensitizers in many cancer cell lines [32-34]. In several preclinical studies it has been shown that combining HDAC inhibitors with TRAIL induces the apoptosis in breast cancer cells, however the exact mechanism of this induction is still not known $[35,36]$. It has been proposed, that the enhancement of this process may be due to upregulation of TRAIL receptors, redistribution of TRAIL receptors, down-regulation of antiapoptotic proteins, and up-regulation of proapoptotic proteins such as Bcl-2 [37]. Given the elevated expression of TRAIL-related apoptosis markers our results together with the findings presented by Chopin et al. and Singh at al. $[35,36]$ may indicate, that concomitant treatment of IORT and HDAC inhibitors is likely to be a benefit in outcome on breast cancer patients.

\section{CONCLUSIONS}

In this paper we have described the activation of two apoptotic pathways: extrinsic and intrinsic, on the transcript level upon incubation of breast cancer MCF7 cell line with wound fluids collected from patients after breast cancer conserving surgery or breast cancer conserving surgery followed by IORT treatment. Our finding provides a valuable data on the activation of extrinsic apoptotic pathway in MCF7 cell line after incubation with wound fluids collected from patients after IORT. We believe that our findings will contribute to better understanding of biological processes involved in response of breast cancer cells to IORT treatment and will be particularly relevant in the effectiveness of breast cancer treatment.

\section{Acknowlegment}

This work was supported by the National Science Centre (grant number: 2015/19/D/NZ5/02190) and the Greater Poland Cancer Centre (grant number: 25/2016[140]).

\section{Disclosure of potential conflicts of interest}

The authors indicate that there is no conflict of interest.

\section{Authors' contributions}

KK was involved in conception and design of the manuscript, performing the experiments, writing the manuscript and revising it critically for important intellectual content, IP was involved in performing the experiments and revising the manuscript, $\mathrm{KZ}$ was involved in performing the experiments, DM was involved in writing the manuscript and revising it critically for important intellectual content, WMS was involved in revising manuscript critically for important intellectual content and given final approval of the version to be published.

\section{REFERENCES}

1. Lipponen P, Aaltomaa S, Kosma VM, et al. Apoptosis in breast cancer as related to histopathological characteristics and prognosis. European Journal of Cancer. 1994; 30(14): 2068-2073, doi: 10.1016/0959_ -8049(94)00342-3.

2. Wang D, Hu K, Gao N, et al. High throughput screening of cytokines, chemokines and matrix metalloproteinases in wound fluid induced by mammary surgery. Oncotarget. 2015; 6(30): 29296-29310, doi: 10.18632/oncotarget.4828, indexed in Pubmed: 26313265.

3. Demicheli R, Valagussa P, Bonadonna G. Does surgery modify growth kinetics of breast cancer micrometastases? Br J Cancer. 2001; 85(4): 490-492, doi: 10.1054/bjoc.2001.1969, indexed in Pubmed: 11506484.

4. Candido J, Hagemann T. Cancer-related inflammation. J Clin Immunol. 2013; 33 Suppl 1: S79-S84, doi: 10.1007/s10875-012-9847-0, indexed in Pubmed: 23225204 
5. Benson JR, Jatoi I, Keisch M, et al. Early breast cancer. Lancet. 2009; 373(9673): 1463-1479, doi: 10.1016/S0140-6736(09)60316-0, indexed in Pubmed: 19394537.

6. Piotrowski I, Kulcenty K, Wichtowski M, et al. Intraoperative Radiotherapy of Breast Cancer and Its Biological Effects. Breast Care (Basel). 2017; 12(2): 109-113, doi: 10.1159/000454673, indexed in Pubmed: 28559768.

7. Belletti B, Vaidya JS, D'Andrea S, et al. Targeted intraoperative radiotherapy impairs the stimulation of breast cancer cell proliferation and invasion caused by surgical wounding. Clin Cancer Res. 2008; 14(5): 1325-1332, doi: 10.1158/1078-0432.CCR-07-4453, indexed in Pubmed: 18316551.

8. Tagliabue E, Agresti R, Carcangiu ML, et al. Role of HER2 in wound-induced breast carcinoma proliferation. Lancet. 2003; 362(9383): 527-533, doi: 10.1016/S0140-6736(03)14112-8, indexed in Pubmed: 12932384.

9. Fidler IJ. The pathogenesis of cancer metastasis: the 'seed and soil' hypothesis revisited. Nat Rev Cancer. 2003; 3(6): 453-458, doi: 10.1038/nrc1098, indexed in Pubmed: 12778135

10. Mothersill C, Seymour C. Radiation-induced bystander effects and adaptive responses--the Yin and Yang of low dose radiobiology? Mutat Res. 2004; 568(1): 121-128, doi: 10.1016/j.mrfmmm.2004.06.050, indexed in Pubmed: 15530545.

11. Hengartner MO. The biochemistry of apoptosis. Nature. 2000;407(6805): 770-776, doi: 10.1038/35037710, indexed in Pubmed: 11048727.

12. Degterev A, Boyce M, Yuan J. A decade of caspases. Oncogene. 2003; 22(53): 8543-8567, doi: 10.1038/sj.onc.1207107, indexed in Pubmed: 14634618 .

13. Kaina B. DNA damage-triggered apoptosis: critical role of DNA repair, double-strand breaks, cell proliferation and signaling. Biochem Pharmacol. 2003; 66(8): 1547-1554, indexed in Pubmed: 14555233.

14. Zaleska K, Przybyła A, Kulcenty K, et al. Wound fluids affect miR-21, miR155 and miR-221 expression in breast cancer cell lines, and this effect is partially abrogated by intraoperative radiation therapy treatment. Oncol Lett. 2017; 14(4): 4029-4036, doi: 10.3892/ol.2017.6718, indexed in Pubmed: 28943910.

15. Livak KJ, Schmittgen TD. Analysis of relative gene expression data using real-time quantitative PCR and the 2(-Delta Delta $C(T)$ ) Method. Methods. 2001; 25(4): 402-408, doi: 10.1006/meth.2001.1262, indexed in Pubmed: 11846609.

16. Ashkenazi A. Targeting death and decoy receptors of the tumour-necrosis factor superfamily. Nat Rev Cancer. 2002; 2(6): 420-430, doi: 10.1038/nrc821, indexed in Pubmed: 12189384

17. Holmgren L, O'Reilly MS, Folkman J. Dormancy of micrometastases: balanced proliferation and apoptosis in the presence of angiogenesis suppression. Nat Med. 1995; 1(2): 149-153, indexed in Pubmed: 7585012.

18. Veldwijk MR, Gerhardt A, Giordano FA, et al. Comparison of the proliferative and clonogenic growth capacity of wound fluid from breast cancer patients treated with and without intraoperative radiotherapy. Translational Cancer Research. 2015; 4: 173-177.

19. Segatto I, Berton $S$, Sonego $M$, et al. Surgery-induced wound response promotes stem-like and tumor-initiating features of breast cancer cells, via STAT3 signaling. Oncotarget. 2014; 5(15): 6267-6279, doi: 10.18632/oncotarget.2195, indexed in Pubmed: 25026286.

20. Makin G, Dive C. Apoptosis and cancer chemotherapy. Trends in cell biology. 2001; 11: S22-26.

21. Keane MM, Ettenberg SA, Nau MM, et al. Chemotherapy augments TRAIL-induced apoptosis in breast cell lines. Cancer Res. 1999; 59(3): 734-741, indexed in Pubmed: 9973225.

22. Singh TR, Shankar S, Chen X, et al. Synergistic interactions of chemotherapeutic drugs and tumor necrosis factor-related apoptosis-inducing ligand/Apo-2 ligand on apoptosis and on regression of breast carcinoma in vivo. Cancer Res. 2003; 63(17): 5390-5400, indexed in Pubmed: 14500373.

23. Wang S, El-Deiry WS. TRAIL and apoptosis induction by TNF-family death receptors. Oncogene. 2003; 22(53): 8628-8633, doi: 10.1038/sj.onc.1207232, indexed in Pubmed: 14634624.

24. Rahman M, Pumphrey JG, Lipkowitz S, et al. The TRAIL to targeted therapy of breast cancer. Adv Cancer Res. 2009; 103: 43-73, doi: 10.1016/S0065-230X(09)03003-6.

25. Ashkenazi A, Pai RC, Fong $S$, et al. Safety and antitumor activity of recombinant soluble Apo2 ligand. J Clin Invest. 1999; 104(2): 155-162, doi: 10.1172/JCl6926, indexed in Pubmed: 10411544.

26. Wang S, El-Deiry WS. Requirement of p 53 targets in chemosensitization of colonic carcinoma to death ligand therapy. Proc Natl Acad Sci U S A. 2003; 100(25): 15095-15100, doi: 10.1073/pnas.2435285100, indexed in Pubmed: 14645705.

27. Chinnaiyan AM, Prasad U, Shankar S, et al. Combined effect of tumor necrosis factor-related apoptosis-inducing ligand and ionizing radiation in breast cancer therapy. Proc Natl Acad Sci U S A. 2000; 97(4): 1754-1759, doi: 10.1073/pnas.030545097, indexed in Pubmed: 10677530.

28. Buchsbaum DJ, Zhou T, Grizzle WE, et al. Antitumor efficacy of TRA-8 anti-DR5 monoclonal antibody alone or in combination with chemotherapy and/or radiation therapy in a human breast cancer model. Clin Cancer Res. 2003; 9(10 Pt 1):3731-3741, indexed in Pubmed: 14506165.

29. Lagadec C, Adriaenssens E, Toillon RA, et al.Tamoxifen and TRAIL synergistically induce apoptosis in breast cancer cells. Oncogene. 2008; 27(10): 1472-1477, doi: 10.1038/sj.onc.1210749, indexed in Pubmed: 17767197.

30. Wagner JM, Hackanson B, Lübbert M, et al. Histone deacetylase (HDAC) inhibitors in recent clinical trials for cancer therapy. Clin Epigenetics. 2010; 1(3-4): 117-136, doi: 10.1007/s13148-010-0012-4, indexed in Pubmed: 21258646.

31. Carew JS, Giles FJ, Nawrocki ST. Histone deacetylase inhibitors: mechanisms of cell death and promise in combination cancer therapy. Cancer Lett. 2008; 269(1): 7-17, doi: 10.1016/j.canlet.2008.03.037, indexed in Pubmed: 18462867.

32. Chinnaiyan $P$, Vallabhaneni $G$, Armstrong $E$, et al. Modulation of radiation response by histone deacetylase inhibition. Int J Radiat Oncol Biol Phys. 2005; 62(1): 223-229, doi: 10.1016/j.jirobp.2004.12.088, indexed in Pubmed: 15850925.

33. Munshi A, Tanaka T, Hobbs ML, et al. Vorinostat, a histone deacetylase inhibitor, enhances the response of human tumor cells to ionizing radiation through prolongation of gamma-H2AX foci. Mol Cancer Ther. 2006; 5(8): 1967-1974, doi: 10.1158/1535-7163.MCT-06-0022, indexed in Pubmed: 16928817

34. Munshi A, Kurland JF, Nishikawa T, et al. Histone deacetylase inhibitors radiosensitize human melanoma cells by suppressing DNA repair activity. Clin Cancer Res. 2005; 11(13): 4912-4922, doi: 10.1158/1078-0432. CCR-04-2088, indexed in Pubmed: 16000590.

35. Chopin V, Slomianny $\mathrm{C}$, Hondermarck $\mathrm{H}$, et al. Synergistic induction of apoptosis in breast cancer cells by cotreatment with butyrate and TNF-alpha, TRAIL, or anti-Fas agonist antibody involves enhancement of death receptors' signaling and requires P21(waf1). Exp Cell Res. 2004; 298(2): 560-573, doi: 10.1016/j.yexcr.2004.04.038, indexed in Pubmed: 15265702.

36. Singh TR, Shankar S, Srivastava RK. HDAC inhibitors enhance the apoptosis-inducing potential of TRAIL in breast carcinoma. Oncogene. 2005; 24(29): 4609-4623, doi: 10.1038/sj.onc.1208585, indexed in Pubmed: 15897906.

37. Fulda S. Modulation of TRAIL-induced apoptosis by HDAC inhibitors. Curr Cancer Drug Targets. 2008; 8(2): 132-140, indexed in Pubmed: 18336196. 